\title{
Movement of Self-Organizing Solitons in Ferromagnet
}

\author{
V. V. Nietz \\ Joint Institute for Nuclear Research, Dubna, Moscow Region, Russia \\ Email: nietz@jinr.ru
}

Received 27 July 2015; accepted 15 September 2015; published 18 September 2015

Copyright (C) 2015 by author and Scientific Research Publishing Inc.

This work is licensed under the Creative Commons Attribution International License (CC BY).

http://creativecommons.org/licenses/by/4.0/

(c) (i) Open Access

\begin{abstract}
Precessing ball solitons (PBS) in a ferromagnet during the first order phase transition induced by a magnetic field directed along the axis of anisotropy, while the additional action of high-frequency field perpendicular to the main magnetic field, are analyzed. It is shown that the spatial motion of solitons, associated with thermal fluctuations in the crystal, does not destroy the equilibrium of self-organized PBS.
\end{abstract}

\section{Keywords}

Ferromagnet, First-Order Phase Transition, Magnetic Field, High-Frequency Magnetic Field, Precessing Ball Soliton, Self-Organizing State, Equilibrium, Spatial Motion of Solitons

\section{Introduction}

Precessing ball solitons (PBS) that may occur at the first order phase transition in a uni-axis ferromagnet under the action of a magnetic field along the easy axis has been considered in several papers [1]-[11]. The main characteristics of PBS have been investigated in articles [10] [11]. In [12] it was shown that with the additional effect of high-frequency magnetic field perpendicular to the main field, there may be of self-organizing state of PBS, when the decrease of the energy associated with dissipation, fully offset by the influx of energy from the source of the high-frequency field. But in this case, only immobile PBS were considered, i.e. without three-dimensional motion. The question of the existence of equilibrium self-organizing states of moving PBS remained open.

In this paper, an analysis was conducted and it was shown that the existence of self-organizing state for PBS had a dynamic character, in which strict observance of the equilibrium was disrupted, but immediately the movement was damped, and thereby the equilibrium state of self-organized PBS restored. i.e. actually selforganizing state for PBS exists even at three-dimensional motion. 


\section{Analysis of PBS Movement}

To analyze magnetic solitons in a ferromagnet at the first-order transition in the presence of periodic magnetic field, as in [12], we use the Landau-Lifshitz equation with a Gilbert form of dissipative term [13]:

$$
\frac{\partial \mathbf{m}}{\partial t}=\frac{2 \mu_{B}}{\hbar} \mathbf{m} \times \frac{\delta W}{\delta \mathbf{m}}+\kappa\left(\mathbf{m} \times \frac{\partial \mathbf{m}}{\partial t}\right)
$$

and corresponding expression for the density of thermodynamic potential (as in [12])

$$
W=\frac{K_{1}}{2}\left|m_{\perp}\right|^{2}+\frac{\alpha}{2}\left[\left(\frac{\partial \mathbf{m}}{\partial X}\right)^{2}+\left(\frac{\partial \mathbf{m}}{\partial Y}\right)^{2}\right]+\frac{\alpha_{z}}{2}\left(\frac{\partial \mathbf{m}}{\partial Z}\right)^{2}-m_{z}\left(H_{z}+4 \pi M_{0}\right)-\left(\mathbf{m H}_{\perp}\right)-\frac{2 \pi}{3}\left(1+m_{z}\right)^{2} M_{0} .
$$

Here $H_{z}>0, \mathbf{H}_{\perp}$ is the periodic field directed perpendicular to the Z-axis and to the main field $H_{z}, K_{1}>0$, $\kappa>0 ; \mathbf{m}$ is a non-dimensional vector of ferromagnetism equal in the absolute value to $1 ; m_{\perp}=m_{x}+i m_{y}$, in initial state: $m_{z}=-1$.

We consider the PBS in a flat plate perpendicular to the Z-axis, use the following dimensionless values: $\tau=2 \mu_{B} K_{1} \hbar^{-1} t, \quad x=K_{1}^{0.5} \alpha^{-0.5} X, \quad y=K_{1}^{0.5} \alpha^{-0.5} Y, \quad z=K_{1}^{0.5} \alpha_{z}^{-0.5} Z ;$ and note: $h=H_{z} / K_{1}+4 \pi M_{0} / K_{1}$,

$D=4 \pi M_{0} / 3 K_{1}$. The change of sign of the self-demagnetizing field for PBS, $E_{d e m, s}=-\frac{2 \pi}{3}\left(1+m_{z}\right)^{2} M_{0}$ in Equation (2), in comparison with article [12], does not lead to a qualitative change in results, but is logically more correct.

If added periodic field is

$$
\mathbf{H}_{\perp}=K_{1} h_{\perp} \mathrm{e}^{\mathrm{i} \omega_{0} \tau},
$$

the Equation (1) can be present in form

$$
i \dot{m}_{\perp}=-(h+D) m_{\perp}-(1+D) m_{z} m_{\perp}+m_{z} \Delta m_{\perp}-m_{\perp} \Delta m_{z}+\kappa\left(m_{\perp} \dot{m}_{z}-m_{z} \dot{m}_{\perp}\right)+h_{\perp} m_{z} \mathrm{e}^{i \omega_{0} \tau} .
$$

In the case if the movement of PBS is directed along Z-axis, i.e. $\vec{k}=k_{z}(\tau)$, the expression for magnetic component is

$$
m_{\perp}(\mathbf{r}, \tau)=p(\mathbf{r}, \tau) \mathrm{e}^{i \phi}=p(r, \tau) \mathrm{e}^{i\left(\omega_{0} \tau-k_{z} r_{z}-\beta(r, \tau)\right)} .
$$

In this paper, the PBS is considered in a spherical coordinate system, when its beginning coincides with the center of the soliton:

$$
m_{\perp}(\rho, \theta, \tau)=p(\rho, \tau) \mathrm{e}^{i\left(\omega_{0} \tau-k_{z} \rho \cos \theta-\beta(\rho, \tau)\right)} .
$$

In this case $r_{z}=\rho \cos \theta$, the phase shift associated with moving becomes zero in the center of the PBS, and phase shift for other parts of the soliton is determined by the values of $\rho$ and the angle $\theta$ between vector $\vec{\rho}$ and Z-axis: $\rho \cos \theta>0$ if $\theta<\pi / 2$, and $\rho \cos \theta<0$ if $\theta>\pi / 2$.

The phase of precession of magnetic moments for localized excitation depends on a radius, i.e. $\beta=\beta(\rho, \tau)$. From (4) equation, we have the next two equations that describe the configuration of the soliton and the time dependence of the parameter $m_{z}(\rho, \tau)$ :

$$
\begin{aligned}
\Delta m_{z}+\frac{m_{z}}{\left(1-m_{z}^{2}\right)}\left(\nabla m_{z}\right)^{2}= & -\left(1-m_{z}^{2}\right)\left[(1+D) m_{z}+\left(h-\omega_{0}+\frac{\partial k_{z}}{\partial \tau} \rho \cos \theta+\frac{\partial \beta}{\partial \tau}+D\right)\right] \\
& +m_{z}\left(1-m_{z}^{2}\right)\left(\vec{k}_{z} \cos \theta \vec{i}_{\rho}-\vec{k}_{z} \sin \theta \vec{i}_{\theta}+\frac{\partial \beta}{\partial \rho} \vec{i}_{\rho}\right)^{2} \\
& +\kappa \frac{\partial m_{z}}{\partial \tau}+h_{\perp} m_{z} \sqrt{1-m_{z}^{2}} \cos \left(k_{z} \rho \cos \theta+\beta\right)
\end{aligned}
$$




$$
\begin{aligned}
\frac{\partial m_{z}}{\partial \tau}= & \nabla\left[\left(1-m_{z}^{2}\right)\left(\vec{k}_{z} \cos \theta \vec{i}_{\rho}-\vec{k}_{z} \sin \theta \vec{i}_{\theta}+\frac{\partial \beta}{\partial \rho} \vec{i}_{\rho}\right)\right] \\
& +\kappa\left(1-m_{z}^{2}\right)\left(\omega_{0}-\frac{\partial k_{z}}{\partial \tau} \rho \cos \theta-\frac{\partial \beta}{\partial \tau}\right)-h_{\perp} \sqrt{1-m_{z}^{2}} \sin \left(k_{z} \rho \cos \theta+\beta\right) .
\end{aligned}
$$

(In these equation, $\boldsymbol{i}_{\rho}$ and $\boldsymbol{i}_{\theta}$ are the unit vectors of spherical coordinate system.)

The (7) and (8) equations correspond to the expressions describing the time dependencies of the density parameter $m_{z}$ and energy $e$ :

$$
\begin{aligned}
\left(\frac{\partial m_{z}}{\partial \tau}\right)_{\text {change }}= & \sqrt{1-m_{z}^{2}}\left(\kappa \sqrt{1-m_{z}^{2}}\left(\omega_{0}-\frac{\partial k_{z}}{\partial \tau} \rho \cos \theta-\frac{\partial \beta}{\partial \tau}\right)-h_{\perp} \sin \left(k_{z} \rho \cos \theta+\beta\right)\right) \\
\left(\frac{\partial e}{\partial \tau}\right)_{\text {change }}= & -\kappa\left(\frac{1}{\left(1-m_{z}^{2}\right)}\left(\frac{\partial m_{z}}{\partial \tau}\right)^{2}+\left(1-m_{z}^{2}\right)\left(\omega_{0}-\frac{\partial k_{z}}{\partial \tau} \rho \cos \theta-\frac{\partial \beta}{\partial \tau}\right)^{2}\right) \\
& +h_{\perp}\left(-\frac{m_{z}}{\sqrt{1-m_{z}^{2}}} \frac{\partial m_{z}}{\partial \tau} \cos \left(k_{z} \rho \cos \theta+\beta\right)+\sqrt{1-m_{z}^{2}}\left(\omega_{0}-\frac{\partial k_{z}}{\partial \tau} \rho \cos \theta-\frac{\partial \beta}{\partial \tau}\right) \sin \left(k_{z} \rho \cos \theta+\beta\right)\right)
\end{aligned}
$$

(It does not show here the gradient of these expressions related to "domestic" parts of these parameters, which are vanish when integrated over the volume of the soliton.)

We see that for unmoving PBS, i.e. if $k_{z}=0$ and $\frac{\mathrm{d} k_{\mathrm{z}}}{\mathrm{d} \tau}=0$, these equations corresponds to a solution for selforganizing state, when $\left(\frac{\partial m_{z}}{\partial \tau}\right)_{\text {change }}=\left(\frac{\partial e}{\partial \tau}\right)_{\text {change }}=0$ and $\frac{\partial \beta}{\partial \tau}=0$ (see [12]). In this case $\kappa \sqrt{1-m_{z}^{2}} \omega_{0}=h_{\perp} \sin \beta_{0}$. For spherical solitons (PBS) at $k_{z} \equiv 0$, the (7) equation for equilibrium state takes the form:

$$
\begin{aligned}
\frac{\partial^{2} m_{z}}{\partial \rho^{2}}+\frac{2}{\rho} \frac{\partial m_{z}}{\partial \rho}+\frac{m_{z}}{1-m_{z}^{2}}\left(\frac{\partial m_{z}}{\partial \rho}\right)^{2}= & -\left(1-m_{z}^{2}\right)\left[(1+D) m_{z}+\left(h-\omega_{0}+D+\frac{\partial \beta_{0}}{\partial \tau}\right)\right] \\
& +h_{\perp} m_{z}\left(1-m_{z}^{2}\right) \cos \beta_{0}-m_{z} \sqrt{1-m_{z}^{2}}\left(\frac{\partial \beta_{0}}{\partial r}\right)^{2}
\end{aligned}
$$

here

$$
\beta_{0}=\arcsin \left(\frac{\kappa \omega_{0}}{h_{\perp}} \sqrt{1-m_{z}^{2}}\right)
$$

(In (11): $m_{z}=-\sqrt{1-p^{2}}$ for $m_{z}<0$, and $m_{z}=+\sqrt{1-p^{2}}$ for $m_{z}>0$.)

However if $k_{z} \neq 0, \frac{\mathrm{d} k_{z}}{\mathrm{~d} \tau} \neq 0$, this equilibrium state of PBS disappears. Let us consider this situation quantitatively. Kinetic energy of PBS

$$
E_{\text {skin }}=L \int \frac{\left(1-m_{z}^{2}\right)}{2}\left(k_{z}+\nabla \beta\right)^{2} \rho^{2} \mathrm{~d} \rho \cong L k_{z}^{2} \int_{0}^{\infty} \frac{\left(1-m_{z}^{2}\right)}{2} \rho^{2} \mathrm{~d} \rho,
$$

where $L=4 \pi \cdot 0.625 \times 10^{12} K_{1}^{-0.5} \alpha \alpha_{z}^{0.5} M_{0}$. For example, at $T=100 \mathrm{~K}$ the average energy of motion of PBS is $E_{\text {skin }}=k T$. This corresponds for PBS at $h=0.998, \omega_{0}=0.01, h_{\perp}=1.5 \times 10^{-5} \quad\left(M_{0}=0.5 \times 10^{14} \frac{\mathrm{eV}}{\mathrm{Oecm}^{3}} \cong 80 \mathrm{Oe}\right.$, $K_{1}=1000$ Oe , $\alpha=\alpha_{z}=3 \times 10^{-10}$ Oecm $^{2}$, see Figure 1 and Figure 2 ) to the average value of the wave vector 


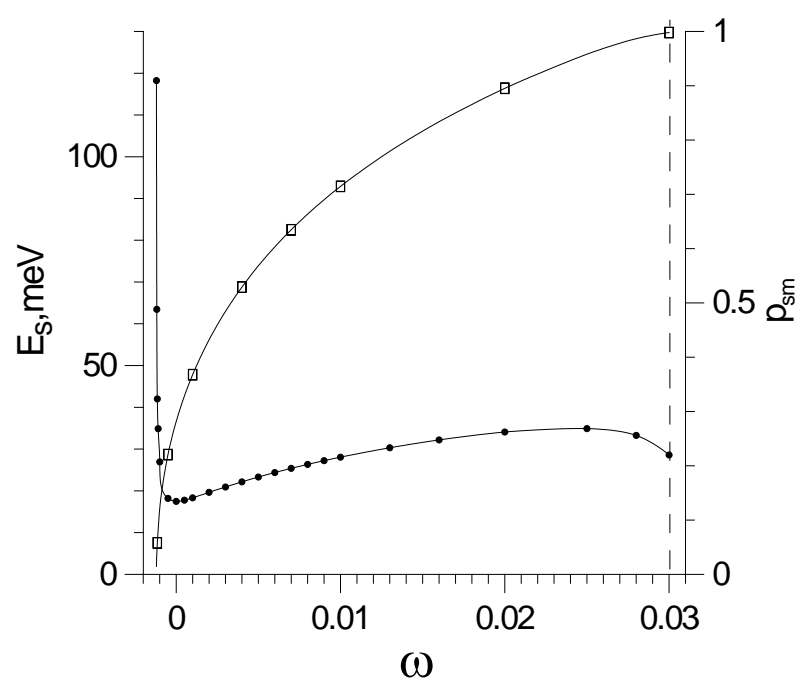

Figure 1. Frequency dependencies of energy and amplitude $p_{s m}$ of PBS for $h=0.998, h_{\perp}=1.5 \times 10^{-5}, \kappa=5 \times 10^{-4}$ (from [12]).

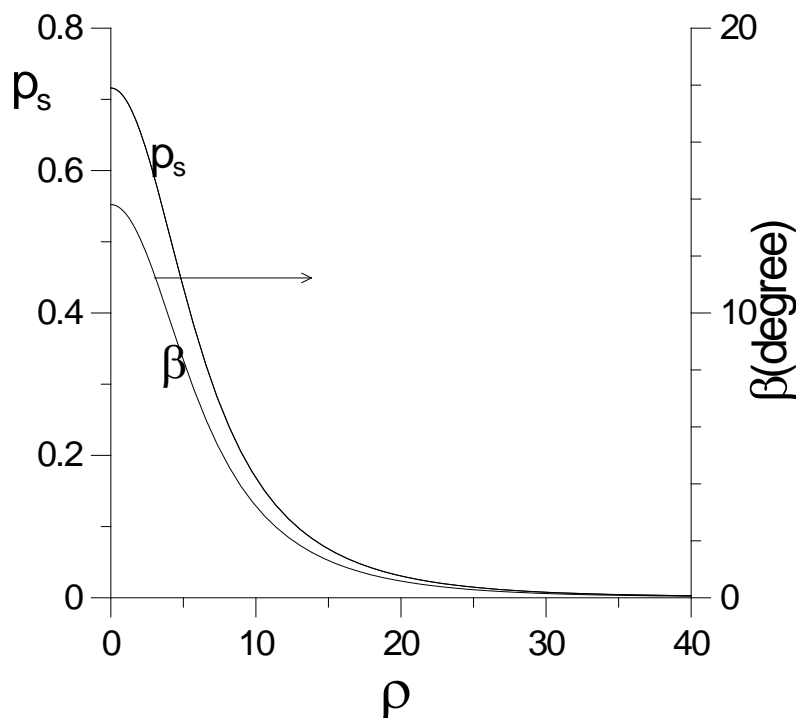

Figure 2. Configuration of equilibrium PBS for $h=0.998, h_{\perp}=1.5 \times 10^{-5}$, $\kappa=5 \times 10^{-4}$, at $\omega_{0}=0.01$, and corresponding dependency of parameter $\beta$ (from [12]).

$k_{z} \cong 1.73$. Consider how parameters of $m_{z}$ and $e$ of equilibrium PBS are changed at occurrence and decreasing of movement. We expand the expression (9) and (10) in powers of the quantities $\frac{\partial k_{z}}{\partial \tau} \rho \cos \theta$ and $k_{z} \rho \cos \theta$, leave only the first degree of decomposition, and believing that at the initial time, at $\tau=0$, all the conditions for equilibrium are fulfilled, we obtain the expression for the change of parameters directly related to the presence of $\frac{\partial k_{z}}{\partial \tau}$ and $k_{z}$ at $\tau>0$ :

$$
\left(\frac{\partial m_{z}}{\partial \tau}\right)_{c h k_{z}}=-\left(\kappa\left(1-m_{z 0}^{2}\right) \frac{\mathrm{d} k_{z}}{\mathrm{~d} \tau}+\sqrt{h_{\perp}^{2}-\kappa^{2} \omega_{0}^{2}\left(1-m_{z 0}^{2}\right)} k_{z}\right) \rho \cos \theta=\left(\frac{\partial m_{z}}{\partial \tau}\right)_{c h 1}+\left(\frac{\partial m_{z}}{\partial \tau}\right)_{c h 2},
$$




$$
\left(\frac{\partial e}{\partial \tau}\right)_{c h k_{z}}=\omega_{0} \sqrt{1-m_{z 0}^{2}}\left(\kappa \sqrt{1-m_{z 0}^{2}} \frac{\mathrm{d} k_{z}}{\mathrm{~d} \tau}+\sqrt{h_{\perp}^{2}-\kappa^{2} \omega_{0}^{2}\left(1-m_{z 0}^{2}\right)} k_{z}\right) \rho \cos \theta=\left(\frac{\partial e}{\partial \tau}\right)_{c h 1}+\left(\frac{\partial e}{\partial \tau}\right)_{c h 2}
$$

There expressions can be split to two parts, proportional to $\frac{\partial k_{z}}{\partial \tau}$ and $k_{z}$ correspondingly. Here is important dependence on the $\theta$.

Excitation of PBS movement due to thermal fluctuations in a crystal are random and chaotic. Evolution of PBS can be presented in two stages. In the first stage, movement due to fluctuations of the kinetic energy arises. In this case $\frac{\partial|k|}{\partial \tau}>0$ and $|k|>0$. For example at $k_{z}>0$, in correspondingly with (14) and (15), there is a deformation of the soliton and redistribution of $m_{z}$ and $e$ densities between the front and the back parts of PBS: at $\cos \theta>0$ there are decreasing of $m_{z}$ but increasing of $e$ - on the front part of PBS, and contrary, increasing of $m_{z}$ but decreasing of $e$ - on the back part of PBS. Of course, strictly speaking, PBS ceases be in equilibrium in this stage. However further, in the second stage of process, restoration of the initial configuration of PBS is carried out, also in accordance with (14) and (15) expressions. In this case $k_{z} \rightarrow 0$, and as a consequence, $\frac{\partial m_{z}}{\partial \tau} \rightarrow 0$ and $\frac{\partial e}{\partial \tau} \rightarrow 0$, i.e. accurate to linear values by $\kappa$ and $h_{\perp}$, equilibrium state of PBS is restored.

$$
\begin{gathered}
\int_{0}^{\pi}\left(\frac{\partial m_{z}(\rho, \theta, \tau)}{\partial \tau}\right)_{c h k_{z}} \mathrm{~d} \theta=\left(\frac{\partial m_{z}(\rho, \tau)}{\partial \tau}\right)_{c^{\prime} k_{z}}=0, \\
\int_{0}^{\pi}\left(\frac{\partial e(\rho, \theta, \tau)}{\partial \tau}\right)_{c^{\prime} k_{z}} \mathrm{~d} \theta=\left(\frac{\partial e(\rho, \tau)}{\partial \tau}\right)_{c^{2} k_{z}}=0 .
\end{gathered}
$$

Note that during the deformation of PBS due moving, and magnetic moment

$$
M_{s}=\frac{L}{K_{1}} \int_{0}^{\infty}\left(1+m_{z}\right) \rho^{2} \mathrm{~d} \rho
$$

and energy $E_{s}=L \int_{0}^{\infty} e(\rho, \tau) \rho^{2} \mathrm{~d} \rho$ where

$$
\begin{gathered}
e(\rho, \tau)=\frac{(1+D)\left(1-m_{z}^{2}\right)}{2}-(h+D) m_{z}+\frac{1}{2\left(1-m_{z}^{2}\right)}\left(\frac{\partial m_{z}}{\partial \rho}\right)^{2} \\
+\frac{\left(1-m_{z}^{2}\right)}{2}\left(\frac{\partial \beta}{\partial \rho}\right)^{2}-h_{\perp} \sqrt{1-m_{z}^{2}} \cos \beta-h-D,
\end{gathered}
$$

without taking into account the kinetic energy (see [12]), do not change.

For $\omega_{0}=0.01$ at $\rho=10$, the value $p \cong 0.15$, i.e. $m_{z} \cong-0.988$ (see Figure 2). In accordance with the expression $\left(\frac{\partial m_{z}}{\partial \tau}\right)_{1}$ in Equation (14), the change $\Delta k_{z}=1.73$ causes a change $\Delta m_{z} \cong-1.9 \times 10^{-4}$, i.e. only to $\cong 10^{-4}$ part of the initial value. Can be estimated the time $\Delta t$ during which this change is carried out at return to the initial state. In accordance with the expression $\left(\frac{\partial m_{z}}{\partial \tau}\right)_{2}$ in Equation (14), taking into account that $h_{\perp}=1.5 \times 10^{-5}, \quad \beta_{0} \cong 2.5^{\circ}$, we receive for $\Delta m_{z} \cong-1.9 \times 10^{-4}: \Delta t \cong 3.8 \times 10^{-3} \mu \mathrm{sc}$.

For $\omega_{0}=0.03$ at $\rho=4$, the value $p \cong 0.6$, i.e. $m_{z} \cong-0.8$ (see Figure 3 ). The change $\Delta k_{z}=0.836$ causes a change $\Delta m_{z} \cong-0.72 \times 10^{-3}$, i.e. only to $\cong 10^{-3}$ part of the initial value. Taking into account that in this case $\beta_{0} \cong 36^{\circ}$, we receive $\Delta t \cong 1.3 \times 10^{-3} \mu \mathrm{sc}$.

\section{Angle Parameter as Invariant of Landau-Lifshitz Equation for Equilibrium PBS}

Let us consider one more invariant of (4) equation, namely density of a momentum: 


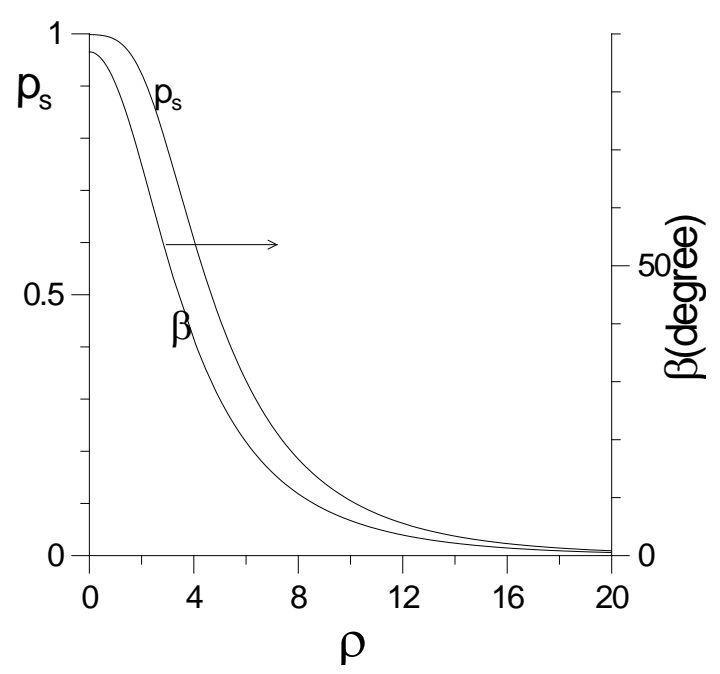

Figure 3. The same as in Figure 2, at $\omega_{0}=0.03$.

$$
\mathbf{p}(\mathbf{r}, \tau)=-\frac{i}{2} \sqrt{\frac{1+m_{z}}{1-m_{z}}}\left(m_{\perp} \nabla m_{\perp}^{*}-m_{\perp}^{*} \nabla m_{\perp}\right) .
$$

Using (6) expression, we obtain for the density of momentum in spherical coordinate system:

$$
\overrightarrow{\mathbf{p}}(\rho, \theta, \tau)=\left(1+m_{z}\right)\left(\vec{k}_{z} \cos \theta \vec{i}_{\rho}-\vec{k}_{z} \sin \theta \vec{i}_{\theta}+\frac{\partial \beta}{\partial \rho} \vec{i}_{\rho}\right) .
$$

This expression refers to the case of moving precessing ball soliton at the presence of high-frequency field when the energy dissipation is almost completely offset by the influx of energy from the source of the highfrequency field. Note that if $\vec{k}_{z}=0$ :

$$
\overrightarrow{\mathbf{p}}(\rho, \tau)=\left(1+m_{z}\right) \frac{\partial \beta}{\partial \rho} \vec{i}_{\rho}
$$

Integrating the expression (21) on the values of the angle $\theta$, we obtain the expression for total momentum of PBS:

$$
\overrightarrow{\mathbf{P}}_{s}(\tau)=\frac{\hbar M_{0}}{2 \mu_{B}} \int \overrightarrow{\mathbf{p}}(\rho, \theta, \tau) \mathrm{d} V=\frac{2 \pi \hbar M_{0}}{\mu_{B}} \int_{0}^{\infty}\left(1+m_{z}\right)\left(\vec{k}_{z}+\frac{\partial \beta}{\partial \rho} \vec{i}_{\rho}\right) \rho^{2} \mathrm{~d} \rho .
$$

Differentiating (21) and using (9), we have

$$
\frac{\mathrm{d} \overrightarrow{\mathbf{p}}(\mathbf{r}, \tau)}{\mathrm{d} \tau}=-\nabla\left[\left(1+m_{z}\right)\left(\omega_{0}-\frac{\mathrm{d} k_{z}}{\mathrm{~d} \tau} \rho-\frac{\partial \beta}{\partial \tau} \vec{i}_{\rho}\right)\right]_{\text {grad }}+\left(\frac{\mathrm{d} \overrightarrow{\mathbf{p}}}{\mathrm{d} \tau}\right)_{\text {change }},
$$

where

$$
\begin{aligned}
\left(\frac{\mathrm{d} \overrightarrow{\mathbf{p}}}{\mathrm{d} \tau}\right)_{\text {change }}= & -\sqrt{1-m_{z}^{2}}\left[\kappa \sqrt{1-m_{z}^{2}}\left(\omega_{0}-\frac{\mathrm{d} k_{z}}{\mathrm{~d} \tau} \rho \cos \theta-\frac{\partial \beta}{\partial \tau} \vec{i}_{\rho}\right)-h_{\perp} \sin \left(k_{z} \rho \cos \theta+\beta\right)\right] \\
& \times\left(k_{z} \cos \theta+\frac{\partial \beta}{\partial \rho} \vec{i}_{\rho}\right)+\frac{\partial m_{z}}{\partial \rho}\left(\omega_{0}-\frac{\partial k_{z}}{\partial \tau} \rho \cos \theta-\frac{\partial \beta}{\partial \tau} \vec{i}_{\rho}\right) .
\end{aligned}
$$

Note that (24) equation also satisfies all the conditions for equilibrium of PBS in self-organizing state, if $k_{z}=\frac{d k_{z}}{d \tau}=0$. 
It is believed that similar to momentum (21), the value $\frac{\partial \beta}{\partial \rho}$ is invariant for the PBS, moving along the axis $z:$

$$
\frac{\partial \beta}{\partial \rho}(\rho, \tau) \vec{i}_{\rho}=\frac{\overrightarrow{\mathbf{p}}(\rho, \theta, \tau)}{\left(1+m_{z}\right)}-\vec{k}_{z}\left(\cos \theta \vec{i}_{\rho}-\sin \theta \vec{i}_{\theta}\right) .
$$

To evaluate the effect of motion on the PBS, we consider value $\frac{\partial \beta}{\partial \tau}$ obtained from (9) and (12). We are using (9) where there is the most direct connection between $m_{z}$ and $k_{z}$. From here, the change in the angular parameter near equilibrium is equal to

$$
\begin{aligned}
\left(\frac{\partial \beta(\rho, \theta, \tau)}{\partial \tau}\right)_{c h k_{z}} & =\frac{\partial}{\partial \tau} \arcsin \left(\frac{\kappa \omega_{0}}{h_{\perp}} \sqrt{1-m_{z}^{2}}\right)=\frac{-\kappa \omega_{0} m_{z 0}}{\sqrt{h_{\perp}^{2}-\kappa^{2} \omega_{0}^{2}\left(1-m_{z 0}^{2}\right)} \sqrt{1-m_{z 0}^{2}}}\left(\frac{\partial m_{z}}{\partial \tau}\right)_{c h k_{z}} \\
& =\frac{\kappa \omega_{0} m_{z 0}}{\sqrt{h_{\perp}^{2}-\kappa^{2} \omega_{0}^{2}\left(1-m_{z 0}^{2}\right)} \sqrt{1-m_{z 0}^{2}}}\left[\kappa\left(1-m_{z 0}^{2}\right) \frac{\partial k_{z}}{\partial \tau}+h_{\perp} \cos \beta_{0} \cdot k_{z}\right] \rho \cos \theta
\end{aligned}
$$

This expression can be split into two parts:

$$
\left(\frac{\mathrm{d} \beta(\rho, \theta, \tau)}{\mathrm{d} k_{z}}\right)_{c h 1}=\frac{\kappa^{2} \omega_{0} m_{z 0} \sqrt{1-m_{z 0}^{2}}}{\sqrt{h_{\perp}^{2}-\kappa^{2} \omega_{0}^{2}\left(1-m_{z 0}^{2}\right)}} \rho \cos \theta
$$

- quantity related with $\frac{\mathrm{d} k_{z}}{\mathrm{~d} \tau}$, and

$$
\left(\frac{\partial \beta(\rho, \theta, \tau)}{\partial \tau}\right)_{c h 2}=\frac{\kappa h_{\perp} \omega_{0} m_{z 0} \cos \beta_{0} k_{z}}{\sqrt{h_{\perp}^{2}-\kappa^{2} \omega_{0}^{2}\left(1-m_{z 0}^{2}\right)} \sqrt{1-m_{z 0}^{2}}} \rho \cos \theta
$$

- quantity related with $k_{z}$. Here values $\beta_{0}$ and $m_{z 0}$ relate to initial equilibrium state, at $\tau=0$.

We see that the changes of $\left(\frac{\mathrm{d} \beta}{\mathrm{d} \tau}\right)_{c h k_{z}}$, as for $\left(\frac{\partial m_{z}}{\partial \tau}\right)_{c h k_{z}}$, is proportional to $\cos \theta$. With an increase in $k_{z}$, if $0<\theta<\pi / 2$, i.e. on front part (fore-part) of soliton, the values $\beta$ are increasing, but decreasing with the identical value on back part of soliton, i.e. for condition $\pi / 2<\theta<\pi$. There is contrary change of $m_{z}$ (see the sign in (14)). Thus, we have:

$$
\int_{0}^{\pi}\left(\frac{\partial \beta(\rho, \theta, \tau)}{\partial \tau}\right)_{c h k_{z}} \mathrm{~d} \theta=0
$$

Note that

$$
\int_{0}^{\infty} \int_{0}^{\pi}\left(\frac{\partial \beta(\rho, \theta, \tau)}{\partial \rho}\right)_{c h k_{z}} \mathrm{~d} \theta \mathrm{d} \rho=0
$$

Moreover, integral value

$$
\mathbf{B} \cong \mathbf{B}_{0}=\int \beta(\rho, \theta) \mathrm{d} \rho \mathrm{d} \theta \mathrm{d} \varphi=4 \pi \int_{0}^{\infty} \beta \rho^{2} \mathrm{~d} \rho=4 \pi\left[1-\frac{\kappa \omega_{0} p_{0 m}}{h_{\perp}} \arcsin \left(\frac{\kappa \omega_{0} p_{0 m}}{h_{\perp}}\right)-\sqrt{1-\frac{\kappa^{2} \omega_{0}^{2} p_{0 m}^{2}}{h_{\perp}^{2}}}\right]
$$

(here $p_{0 m}$ is amplitude of PBS) in equilibrium state of PBS does not change.

Therefore, self-organizing state of PBS does not disappear, but continues to live, in spite of the thermal vibra- 
tions in the crystal.

Like to consideration for $\left(\frac{\partial m_{z}}{\partial \tau}\right)_{c^{\prime} k_{z}}$ and $\left(\frac{\partial e}{\partial \tau}\right)_{c_{h k_{z}}}$, we calculate the estimate for $\left(\frac{\partial \beta}{\partial \tau}\right)_{c h k_{z}}$. For $\omega_{0}=0.01$ at $\rho=10$, where $m_{z} \cong-0.988$, in accordance with Equation (28), the change $\Delta k_{z}=1.73$ causes a change $\Delta \beta=0.025^{\circ}$. Note that in this case initial value is $\beta_{0} \cong 2.5^{\circ}$. The time required to return from $\left(\beta_{0}+\Delta \beta\right)=2.525^{\circ}$ to the initial value $\beta_{0}=2.5^{\circ}$, in accordance with Equation (29), equals $\Delta t \cong 2.8 \times 10^{-4} \mu \mathrm{sc}$.

For $\omega_{0}=0.03$ at $\rho=4$, where $m_{z} \cong-0.8$, the change $\Delta k_{z}=0.84$ causes a change $\Delta \beta \cong 0.015^{\circ}$. Taking into account that in this case $\beta_{0}=36^{\circ}$, we receive for $\Delta \beta \cong 0.015^{\circ}: \Delta t \cong 0.45 \times 10^{-3} \mu \mathrm{sc}$.

\section{Conclusions}

The analysis of moving precessing ball solitons, arising at the first-order phase transition in ferromagnet at the combined action of main field, causes the transition, and transverse high-frequency magnetic field.

It is shown that the movement caused by thermal fluctuations in a crystal causes a small change in the parameters of solitons and thereby to a very small distortion of equilibrium self-organizing state of PBS. However, this distortion is eliminated very quickly and verily equilibrium state is restored. In practice, equilibrium selforganizing PBS, when dissipative losses of energy are compensated by the influx of energy from the highfrequency field, continues to exist at mechanical motion of PBS inside the crystal.

\section{References}

[1] Dzyaloshinskiy, I.E. and Ivanov, B.A. (1979) Localized Topological Solitons in Ferromagnets. JETP, 29, $592-595$.

[2] Ivanov, B.A. and Kosevich, A.M. (1977) Bound-States of a Large Number of Magnons in the Ferromagnet at the OneIon Anisotropy. JETP, 72, 2000-2015.

[3] Kosevich, A.M., Ivanov, B.A. and Kovalev, A.S. (1981) Dynamic and Topological Solitons in a Ferromagnet. Physica D: Nonlinear Phenomena, 3, 363-373. http://dx.doi.org/10.1016/0167-2789(81)90140-8

[4] Baryakhtar, I.V. and Ivanov, B.A. (1983) Vector Field Dynamic Solitons. Physics Letters A, 98, 222-226. http://dx.doi.org/10.1016/0375-9601(83)90857-5

[5] Ivanov, B.A., Kosevich, A.M. and Kovalev, A.S. (1988) Non-Linear Waves of Magnetization. Dynamic and Topological Solitons. Kiev, Naukova Dumka.

[6] Ivanov, B.A., Kosevich, A.M. and Kovalev, A.S. (1990) Magnetic Solitons. Physics Reports, 194, 119-238.

[7] Nietz, V.V. (2001) Neutron Scattering by Ball Solitons. In Euro-Asian Symposium “Trends in Magnetism”, v. 92 of Physics of Metals and Metallography, Ekaterinburg, Russia, February-March, S243.

[8] Nietz, V.V. (2003) Neutron Scattering by Magnetic Ball Solitons. Journal of Magnetism and Magnetic Materials, 266, 258-267. http://dx.doi.org/10.1016/S0304-8853(03)00244-0

[9] Nietz, V.V. and Osipov, A.A. (2008) Ball Solitons and Kinetics of the First Order Phase Transition. Crystallography Reports, 53, 266-270. http://dx.doi.org/10.1134/S1063774508020156

[10] Nietz, V.V. and Osipov, A.A. (2008) Ball Solitons in Kinetics of the First Order Magnetic Phase Transition. Journal of Magnetism and Magnetic Materials, 320, 1464-1471. http://dx.doi.org/10.1016/j.jmmm.2007.12.002

[11] Nietz, V. (2012) Kinetics of Precessing Ball Solitons in Ferromagnetic at the First-Order Transition. European Physics Journal B, 85, 133. http://arXiv.org/abs/1005.2054

[12] Nietz, V. (2013) Precessing Ball Solitons as Dissipative Structures during a Phase Transition in a Ferromagnet. Applied Mathematics, 4, 78-83. http://arXiv.org/abs/1105.1807v1

[13] Landau, L.D. and Lifshitz, E.M. (1935) On the Theory of the Dispersion of Magnetic Permeability in Ferromagnetic Bodies. Phys. Z. Sowjetunion, 8, 153-164. 